\title{
Comparative Genomics Identifies the Mouse Bmp3 Promoter and an Upstream Evolutionary Conserved Region (ECR) in Mammals
}

\author{
Jonathan W. Lowery, Anna W. LaVigne, Shoichiro Kokabu, Vicki Rosen*
}

Department of Developmental Biology, Harvard School of Dental Medicine, Boston, Massachusetts, United States of America

\begin{abstract}
The Bone Morphogenetic Protein (BMP) pathway is a multi-member signaling cascade whose basic components are found in all animals. One member, BMP3, which arose more recently in evolution and is found only in deuterostomes, serves a unique role as an antagonist to both the canonical BMP and Activin pathways. However, the mechanisms that control BMP3 expression, and the cis-regulatory regions mediating this regulation, remain poorly defined. With this in mind, we sought to identify the Bmp3 promoter in mouse (M. musculus) through functional and comparative genomic analyses. We found that the minimal promoter required for expression in resides within $0.8 \mathrm{~kb}$ upstream of $\mathrm{Bmp} 3$ in a region that is highly conserved with rat ( $R$. norvegicus). We also found that an upstream region abutting the minimal promoter acts as a repressor of the minimal promoter in HEK293T cells and osteoblasts. Strikingly, a portion of this region is conserved among all available eutherian mammal genomes (47/47), but not in any non-eutherian animal (0/136). We also identified multiple conserved transcription factor binding sites in the Bmp3 upstream ECR, suggesting that this region may preserve common cisregulatory elements that govern Bmp3 expression across eutherian mammals. Since dysregulation of BMP signaling appears to play a role in human health and disease, our findings may have application in the development of novel therapeutics aimed at modulating BMP signaling in humans.
\end{abstract}

Citation: Lowery JW, LaVigne AW, Kokabu S, Rosen V (2013) Comparative Genomics Identifies the Mouse Bmp3 Promoter and an Upstream Evolutionary Conserved Region (ECR) in Mammals. PLoS ONE 8(2): e57840. doi:10.1371/journal.pone.0057840

Editor: Bernd Sokolowski, University of South Florida, United States of America

Received November 6, 2012; Accepted January 26, 2013; Published February 22, 2013

Copyright: (c) 2013 Lowery et al. This is an open-access article distributed under the terms of the Creative Commons Attribution License, which permits unrestricted use, distribution, and reproduction in any medium, provided the original author and source are credited.

Funding: This work was supported by: an American Recovery \& Reinvestment Act Award (VR); the Harvard School of Dental Medicine Dean's Scholars Award (JWL and SK); and the Harvard College Research Program Award (AWL). The funders had no role in study design, data collection and analysis, decision to publish, or prparation of the manuscript.

Competing Interests: The authors have declared that no competing interests exist.

*E-mail: vicki_rosen@hsdm.harvard.edu

\section{Introduction}

The Bone Morphogenetic Protein (BMP) pathway is a signaling cascade that has ancient origins in the evolution of animals, arising 1.2-1.4 billion years ago [1,2]. Canonical BMP signaling occurs through BMP ligand interaction with a complex of type I and type II BMP receptors, leading to activation of a class of downstream transcription factors (SMADs in vertebrates, MAD in Drosophila, SMA in C. elegans). Strikingly, this basic mechanism is highly conserved across all animals [2] and, as no non-animal counterparts have been identified, the BMP pathway is likely a key advancement in the evolution of animals.

Although the first observation of BMP activity in mammals was its ability to induce ectopic bone formation [3], BMP signaling has since been implicated in the development of nearly all vertebrate organs and is required for some of the earliest developmental processes, including gastrulation and axis determination $[4,5]$. Thus, it is not surprising that BMP signaling is tightly regulated at many levels. For instance, extracellular antagonists that sequester BMP ligands away from BMP receptors (eg, Noggin) and E3ubiquitin ligases (eg, SMURF1) that promote degradation of BMP receptors and SMADs [6,7] are ancestral mechanisms for reducing BMP pathway activation that are conserved as early as sponges [2].
Arising more recently in evolution, the BMP ligand BMP3 serves a unique function by antagonizing the canonical BMP and Activin pathways. Homologs of BMP3 have only been identified in deuterostomes, but are present as early as echinoderms (sea urchin [8]) and hemichordates (acorn worm (Acorn Worm Genome Project, Baylor). Though the mature domains of the prototypical BMP ligands BMP2/4 and BMP5/6/7 (Dpp and Gbb in Drosophila, respectively) share dramatic identity, BMP3 is highly divergent from other BMP ligands in that it falls into an intermediate phylogenetic clade between TGF- $\beta$ /Activin and BMP ligands $[9,10]$ and shares only $40 \%$ amino acid identity with the ancestral BMP2/4 and BMP5/6/7 groups [11]. For many years after its identification, mammalian BMP3 was thought to function like a typical BMP ligand [12,13]. However, more recent in vivo analyses suggest that BMP3 serves an inhibitory function. For instance, while BMP ligands promote osteogenesis [14], Bmp3 knockout mice have high bone mass, indicating that BMP3 acts as a negative regulator of osteogenesis in vivo [15]. Moreover, BMP3 inhibits BMP2-induced differentiation of osteoprogenitors into osteoblasts, the cells which produce bone matrix $[15,16,17,18]$. These findings have been extended to overexpression studies in chick [19], Xenopus [20,21], and mouse [22], all of which consistently indicate that BMP3 negatively regulates the BMP and Activin pathways. While the precise mechanism for this inhibition remains unclear, BMP3 has been demonstrated to both 
sequester BMP receptors into inactive signaling complexes [15,20] through high affinity interaction with the receptor ACVR2B $[17,23,24]$ and lead to altered TGF- $\beta /$ Activin signaling $[15,16,24,25,26]$, which commonly antagonizes BMP-mediated effects $[27,28,29,30,31,32,33,34,35,36,37,38]$.

Despite significant progress in distinguishing BMP3 as a unique inhibitory ligand among BMPs, the mechanisms that regulate BMP3 expression are unclear. For instance, BMP3 exhibits a restricted expression pattern in vivo $[17,19,25,39,40,41,42,43,44,45,46,47,48,49,50,51,52,53,54]$ and its expression is modulated by several pathways $[25,41,52,55,56,57,58,59,60]$, yet the cis-regulatory elements mediating these effects remain largely unknown. With this goal in mind, we sought to identify the Bmp3 promoter in mouse ( $M$. musculus) through functional and comparative genomic analyses. Having found that the minimal promoter resides within $0.8 \mathrm{~kb}$ upstream of $B m p 3$ in M. musculus, we also identified a highly conserved element (ECR) upstream of the homologous Bmp3 locus in every available eutherian mammal genome but not in any noneutherian animal. We determined the minimal ECR that is present in all reference eutherian mammal genomes and identified the transcription factor binding sites conserved between $M$. musculus, rat ( $R$. norvegicus), and human (H. sapiens). Collectively, our findings suggest that the high level of conservation of the Bmp3 upstream ECR may preserve common cis-regulatory elements that govern $B m p 3$ expression across eutherian mammals.

\section{Materials and Methods}

\section{Plasmid construction}

A series of plasmids containing fragments from the region upstream of Bmp3 in M. musculus were generated from M. musculus genomic DNA using primer pairs as detailed in Table S1. For identification of the $B m p 3$ minimal promoter, genomic fragments contained the first $63 \mathrm{nt}$ of $\mathrm{Bmp} 3$ exon 1 in order to include the annotated $B m p 3$ transcription start site. For directional cloning into pGL4.14 (Promega), which is a promoter-less plasmid that encodes firefly luciferase, or pGL4.26 (Promega), in which firefly luciferase is under the control of the herpes simplex virus Thymidine Kinase minimal promoter, 5' XhoI and $3^{\prime}$ HindIII restriction enzyme cut sites were appended to the genomic fragment by PCR. For directional cloning into pJL114, in which firefly luciferase is controlled upstream of the $B m p 3$ minimal promoter in pJL114, 5' SacI and $3^{\prime} X h o I$ restriction enzyme cut sites were appended to the genomic fragment by PCR. Ligation was performed using DNA Ligation Kit (Takara) at $16^{\circ} \mathrm{C}$ for thirty minutes and transformed into OneShot TOP10 E. coli (Invitrogen) using the manufacturers' protocol.

\section{Cell culture and in vitro experiments}

HEK293T, UMR-106, and C2C12 cells were obtained from ATCG; primary calvarial osteoblasts were isolated from newborn wild type mice as described by Owen \& Pan [61]. All cells were maintained in DMEM GlutaMAX (Gibco) supplemented with 10\% FBS (Gibco).

For RT-PGR analyses, cells were scraped into PBS, centrifuged for $5 \mathrm{~min}$ at $500 \mathrm{x} \mathrm{g}$ at $4^{\circ} \mathrm{C}$, the PBS aspirated, then cells were lysed and RNA collected using the RNeasy Mini Kit (QIAGEN) according to the manufacturer's protocol. cDNA was synthesized using the Transcriptor First Strand cDNA Synthesis Kit (Roche) according to the manufacturer's protocol. Newborn mouse hind limb RNA (collected as per animal protocol \#04043 issued to VR with approval by the Harvard Medical Area Institutional Animal Care and Use Committee) served as a positive control. PCR on
cDNA was performed using One Taq polymerase (NEB) according to the manufacturer's protocol. RT-PCR primers were designed to be complementary to $M$. musculus, $R$. norvegicus, and $H$. sapiens and cross exon boundaries (Bmp3: 5'-GGGTCTATGACAGGTACAGG-3' and 5'-CTTTGGCATGGGGAAGTGGGA-3', Hprt: 5'-CGTGCTGGATTACATTAAAGCACTG-3' and 5'GTCAAGGGCATATCGAACAACAAAC-3').

Luciferase activity was assayed using the Dual-Glo Luciferase Assay System (Promega). Cells were seeded at $3 \mathrm{k} /$ cells per well in a 96-well plate. The next day, a plasmid encoding Renilla luciferase (pGL4.73, Promega) and test plasmids driving firefly luciferase were co-transfected into cells using XtremeGENE (Roche). After 48-hours, firefly and Renilla luciferase activity was quantified using a luminometer (LumiCount, Packard); expression of firefly luciferase under the control of the GMV promoter served as a positive control. Experiments were performed in triplicate or greater and are expressed as mean $\pm^{\prime \prime \prime}$ SEM firefly luciferase/Renilla luciferase ratio. Data were normalized to the promoter-less (pGL4.14) or Thymidine Kinase minimal promoter (pGL4.26) control firefly luciferase plasmids.

\section{In silico experiments}

DNA sequences were aligned using BLASTN [62] Version 2.2.26 \pm or ECR Browser [63] through the respective online servers or locally using MUSCLE in MEGA5 software [64]. Accession number and region of DNA used for these analyses are denoted in the text and/or tables/figures. All analyses were performed between June and August 2012 using database versions current to that time period. The consensus Bmp3 upstream minECR was constructed using the Los Alamos National Laboratory's Simple Consensus Maker (http://www.hiv.lanl. gov/content/sequence/CONSENSUS/consensus.html) using "Output aligned" parameter. For identification of transcription factor binding sites, DNA sequences were first aligned using zPicture [65] then transferred to rVista 2.0 [66]. Transcription factor affinity prediction was performed using TRAP $[67,68]$ using "transfac_2010.1 vertebrates" matrix, "mouse_promoters" background model, and Benjamini-Hochberg multiple test correction. DNA repeat motifs were identified using EMBL-EBI's CENSOR database [69] using the parameter "Mammalian." Unless otherwise noted, all analyses were carried out using the default parameters.

\section{Statistical Analysis}

Statistical significance was determined by One-way ANOVA with post hoc Newman-Keuls correction for multiple pairwise comparisons using GraphPad Prism. A $p$ value of $<0.05$ was considered significant.

\section{Results}

\section{Conservation analysis of the $\mathrm{Bmp} 3$ upstream region}

To identify the M. musculus Bmp3 promoter, we first used ECR Browser [63] to analyze the regions of high nucleotide conservation upstream of the $B m p 3$ transcription start site between $M$. musculus and the closely related $R$. norvegicus. This revealed a high level of nucleotide identity ( $\geq 75 \%$ across sliding $100 \mathrm{nt}$ window) in the approximately $1.9 \mathrm{~kb}$ region proximal to the annotated transcription start site of M. musculus Bmp3 (Figure 1). Within this region, there are two large blocks of $\geq 80 \%$ identity: a proximal one spanning from positions -1 to -806 and a distal one spanning positions -1057 to -1945 . Each of these also contains a smaller region of $\geq 90 \%$ identity: -1 to -167 and -1408 and -1571 , respectively. The nucleotide identity between $M$. musculus 


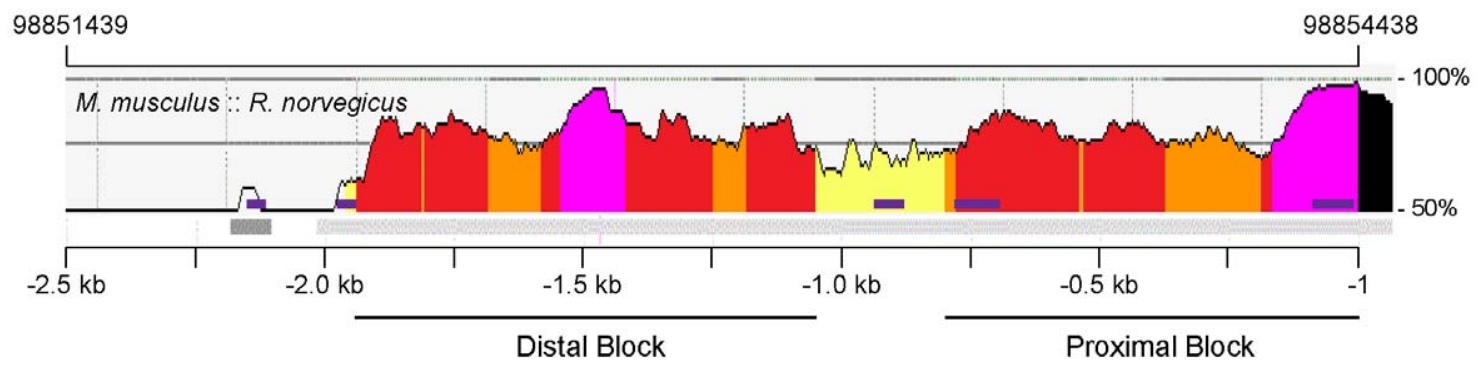

$\%$ Identity per $100 \mathrm{nt}$

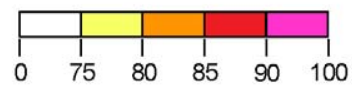

Figure 1. Conservation of the genomic region upstream of Bmp3 in M. musculus and $R$. norvegicus. A: Composite image adapted from ECR Browser aligning the regions upstream of the $B m p 3$ transcription start site between $M$. musculus and $R$. norvegicus. Threshold was set to $\geq 75 \%$ identity across sliding $100 \mathrm{nt}$ window. This revealed a highly conserved $1.9 \mathrm{~kb}$ region proximal to the annotated transcription start site of $M$. musculus $B m p 3$. Within this region, there are two large blocks of $\geq 80 \%$ identity: a proximal one spanning from positions -1 to -806 and a distal one spanning positions -1057 to -1945 . Each of these also contain a smaller region of $\geq 90 \%$ identity: -1 to -167 and -1408 and -1571 , respectively. Genomic coordinates refer to location on M. musculus NC_000071.6. Gray bars indicate aligned segment. Purple boxes indicate repeat elements in M. musculus. Black region indicates $5^{\prime}$-end of $B m p 3$ exon 1 in $M$. musculus. doi:10.1371/journal.pone.0057840.g001

and $R$. norvegicus drops sharply upstream of this region, becoming more conserved once again beyond $3.2 \mathrm{~kb}$ upstream; this poorly conserved region is also present when aligning $M$. musculus and the alternate $R$. norvegicus reference genome, indicating this finding is not due to an assembly error (JWL, data not shown).

\section{Functional identification of the M. musculus $\mathrm{Bmp} 3$ promoter and an upstream repressive element}

Due to the close evolutionary-relatedness of $M$. musculus and $R$. norvegicus, we predicted that the M. musculus Bmp3 promoter would reside within the conserved region that abuts the $B m p 3$ transcription start site instead of farther upstream in the poorly conserved region. We established a reporter system using HEK293T cells wherein firefly luciferase expression is controlled by fragments from the putative $M$. musculus Bmp3 promoter. We first confirmed that HEK293T cells express Bmp3 basally (Figure S1A), making them a suitable system in which to study the $B m p 3$ promoter. Driving firefly luciferase expression by increasingly larger fragments of the putative $B m p 3$ promoter demonstrated that the proximal, highly conserved block $0.800 \mathrm{~kb}$ upstream of $\mathrm{Bmp3}$ is the minimal region necessary for expression (Figure 2A). To evaluate the potential action of this genomic region in osteoblasts, we utilized the osteoblast-like UMR-106 osteosarcoma cell line [70] and primary mouse calvarial osteoblasts, both of which express Bmp3 basally (Figure S1B-C and [60]). Consistent with our findings in HEK293T cells, the $0.800 \mathrm{~kb}$ upstream of $\mathrm{Bmp} 3$ is sufficient to drive firefly luciferase expression in these cells (Figure 2B-C). Specific promoter activity of the $0.800 \mathrm{~kb}$ region was demonstrated by its inability to drive firefly luciferase expression in C2C12 myoblast cells (Figure 2D), which do not express Bmp3 (Figure S1D). rVista2.0 [66] analysis of this $800 \mathrm{nt}$ region (hereafter referred to as the $B m p 3$ minimal promoter) identified binding sites for a number of general (eg, TFII-I) and pathway-specific transcription factors (eg, SMAD, TCF/LEF, AP1, STAT, and KF-kappaB), many of which are conserved with $R$. norvegicus (Table S2). We then performed TRanscription factor Affinity Prediction (TRAP) analyses $[67,68]$ to examine each predicted site based upon strength of binding affinity (Table S2).

Having identified the minimal promoter for M. musculus Bmp3, we turned our attention to the distal conserved block and intervening sequence (IvS) between the distal and proximal blocks
(Figure 1 and Figure 2A). We did not observe promoter activity when attempting to drive firefly luciferase expression with the highest conserved portion of the distal block (Figure 2A). Rather, appending the distal block and IvS to the $B m p 3$ minimal promoter reduced the promoter activity (Figure 2A), indicating that a portion of this $\sim 1 \mathrm{~kb}$ region upstream of the minimal promoter acts as a basal repressive element in HEK293T cells. In support of this finding, rVista 2.0 analysis identifies binding sites for a number of potential repressive transcription factors (Table S2). To determine if the repressive action of this region is specific to HEK293T cells, we examined its function in UMR-106 cells, primary mouse calvarial osteoblasts, and C2C12 cells. In each cell type, the $\sim 1 \mathrm{~kb}$ region upstream of the $B m p 3$ minimal promoter acted as a repressive element, though this did not reach statistical significance in primary osteoblasts (Figure 2B-D).

\section{Identification of an evolutionary conserved region (ECR) upstream of $B m p 3$ in mammals}

The high degree of identity in the distal block/IvS between $M$. musculus and $R$. norvegicus raises the possibility that this could be an evolutionary conserved region (ECR) that regulates the expression of Bmp3. However, the overall level of conservation between $M$. musculus and $R$. norvegicus is too high to allow us to make this conclusion. For this reason, we extended our nucleotide conservation analysis by performing pairwise alignments of the $B m p 3$ upstream regions between $M$. musculus and more distantly related species. We were unable to use ECR Browser for these analyses as the species that are aligned to $M$. musculus in this database are limited. Instead, we performed alignments using BLASTN in sequential $500 \mathrm{nt}$ sections from $M$. musculus against the full-length $5 \mathrm{~kb}$ region upstream of $B m p 3$ from the other species. This approach also allowed for the possibility of genomic insertions or deletions. To ensure the fidelity and accuracy of these analyses, we focused our attention on the thirty-nine NCBI Reference Sequence (RefSeq) animal genomes, at least twenty-eight of which contain an annotated Bmp3 or Bmp3-like gene.

As proof of principle, the highest identity to $M$. musculus was found among the other two muroideans, $R$. norvegicus and Chinese hamster (C. griseus) (Figure 3A and Table S3). Strikingly, among mammals more distantly related to $M$. musculus, we found the highest degree of conservation when using a portion of the distal 
A

M. Musculus Bmp3 Locus

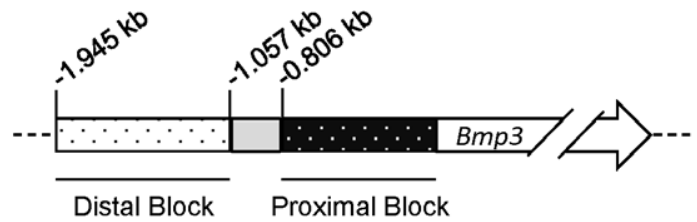

Promoter-less Control

$-0.157 \mathrm{~kb}$

$-0.411 \mathrm{~kb}$

$-0.623 k b$

$-0.800 \mathrm{~kb}$

$-1.797 \mathrm{~kb}$ to $-1.057 \mathrm{~kb}$

$-1.797 \mathrm{~kb}$
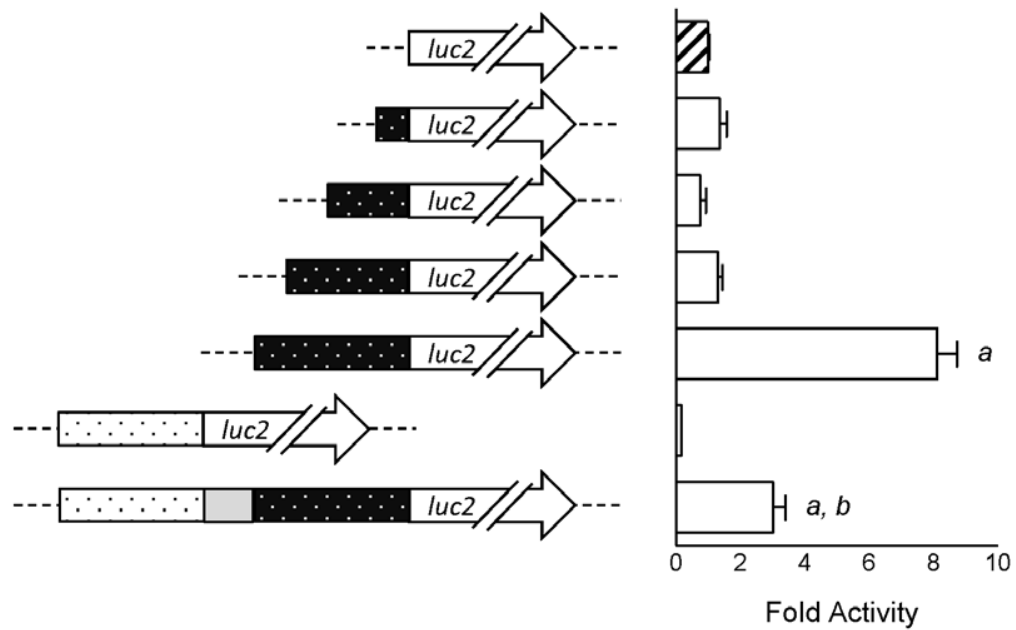

B

UMR-106

C
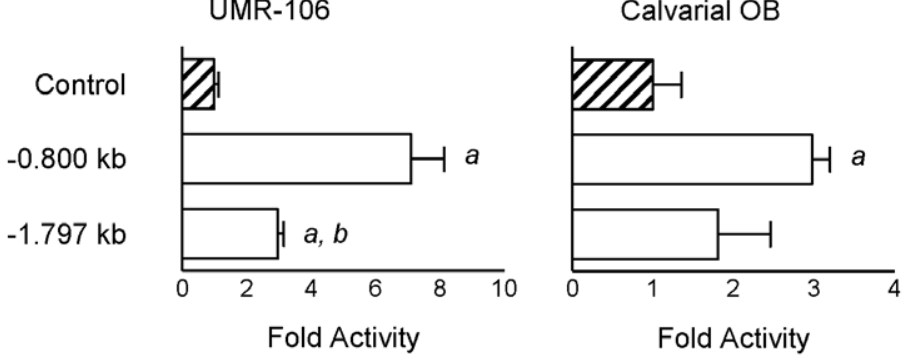

$\mathrm{C} 2 \mathrm{C} 12$

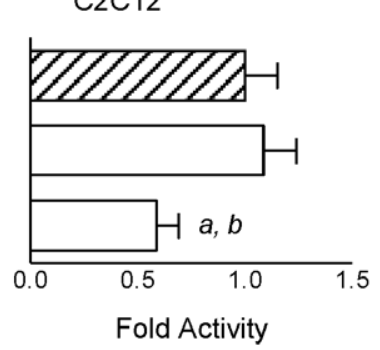

Figure 2. Functional characterization of the genomic region upstream of $M$. musculus Bmp3. A-D: Regulation of firefly luciferase activity driven by fragments from the region upstream of M. musculus Bmp3 in HEK293T cells (A), UMR-106 cells (B), primary calvarial osteoblasts (C), and C2C12 cells (D). All data are mean \pm SEM normalized to promoter-less control. $p<0.05$ as determined by One-way ANOVA with post hoc NewmanKeuls correction is indicated by " $a$ " versus promoter-less control and by "b" versus $-0.800 \mathrm{~kb}$ fragment. doi:10.1371/journal.pone.0057840.g002

conserved block upstream of Bmp3 in M. musculus (Figure 3A-C and Table S3). This pattern was present in every eutherian mammal in our cohort (15/15), but in neither of the non-eutherian mammals, $M$. domestica and O. anatinus (Figure 3D and Table S3), nor in any of the twenty-two non-mammalian RefSeq genomes (JWL, data not shown).

These findings suggested that all or a portion of the distal block conserved between $M$. musculus and R. norvegicus upstream of Bmp3 is an ECR among eutherian mammals. To test this directly, we first aligned the region upstream of Bmp3 in M. musculus and $H$. sapiens using ECR Browser [63]. Setting our threshold at $77 \%$ identity across a sliding 350 nt window to pinpoint lengthy, highly conserved "CoreECRs" [63], we identified a 505 nt region in $M$. musculus spanning from position -1642 to -1138 upstream of the Bmp3 transcription start site that is within the distal block conserved between $M$. musculus and $R$. norvegicus (Figure 4A). The absolute position of the CoreECR is 98852797-98853301 on NG_000071.6. This is the only CoreECR conserved between $M$. musculus and $H$. sapiens within $7.6 \mathrm{~kb}$ upstream and $30 \mathrm{~kb}$ downstream of the Bmp3 locus (JWL, data not shown).
To determine the function of the Bmp3 CoreECR, we generated plasmids in which portions of the repressive distal block/IvS region were placed upstream of the $B m p 3$ minimal promoter. This revealed that the CoreECR is as effective as the complete $\sim 1 \mathrm{~kb}$ region in its ability to repress the $B m p 3$ minimal promoter (Figure 4B).

We then used BLASTN to align the M. musculus: H. sapiens CoreECR sequence to all thirty-nine available animal RefSeq genomes, revealing significant conservation of this sequence in all (15/15) eutherian mammals (Table S4); in each, the conserved region was upstream of $B m p 3$. Moreover, although the $B m p 3$ gene has been found in at least eleven of the non-eutherian animals in the RefSeq genome database, the $B m p 3$ upstream ECR was not found in any of the twenty-four non-eutherian animal RefSeq genomes analyzed (JWL, data not shown).

\section{Determination of the minimal Bmp3 upstream ECR (minECR)}

We have demonstrated that an ECR shared with $M$. musculus lies upstream of $B m p 3$ in each eutherian mammal in the RefSeq genome database. However, the total length and identity of the 
A

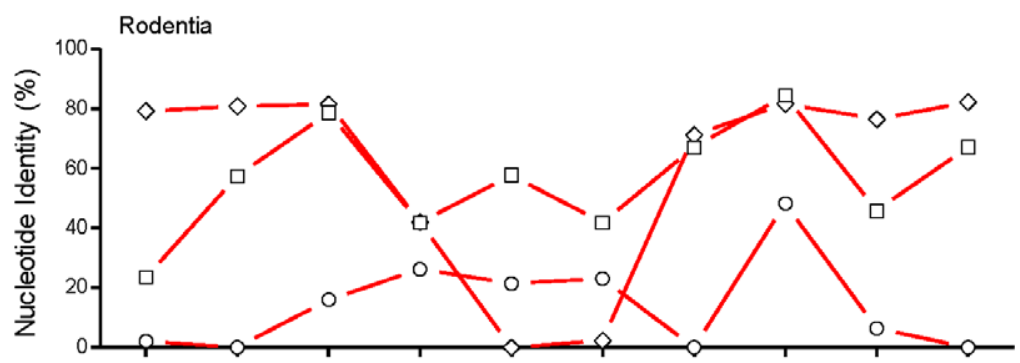

$\diamond \quad R$. norvegicus (Norway rat)

- C. porcellus (Guinea pig)

C. griseus (Chinese hamster)

B

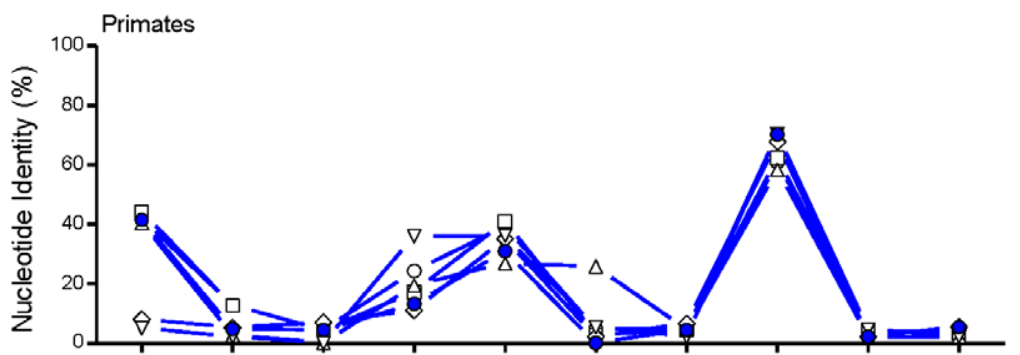

- H. sapiens (Human)



C

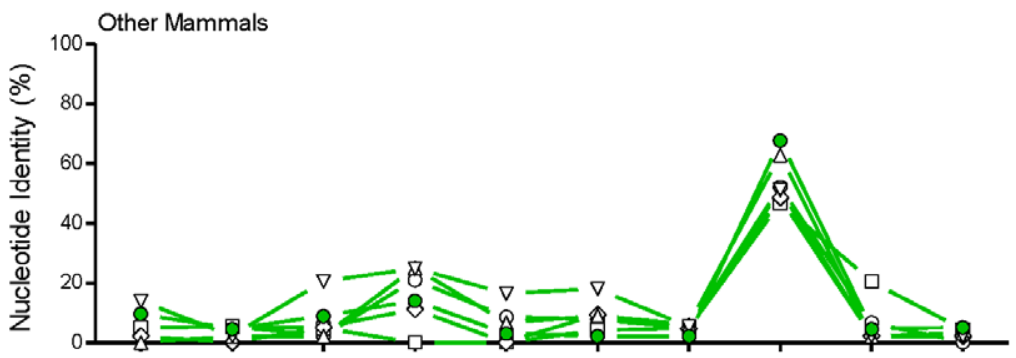

$P$. troglodytes (Chimpanzee)

$\diamond \quad P$. abelii (Orangutan)

$\triangle$ M. mulatta (Rhesus monkey)

$\nabla \quad$ C. jacchus (Marmoset)

- N. leucogenys (White-cheeked gibbon)

D
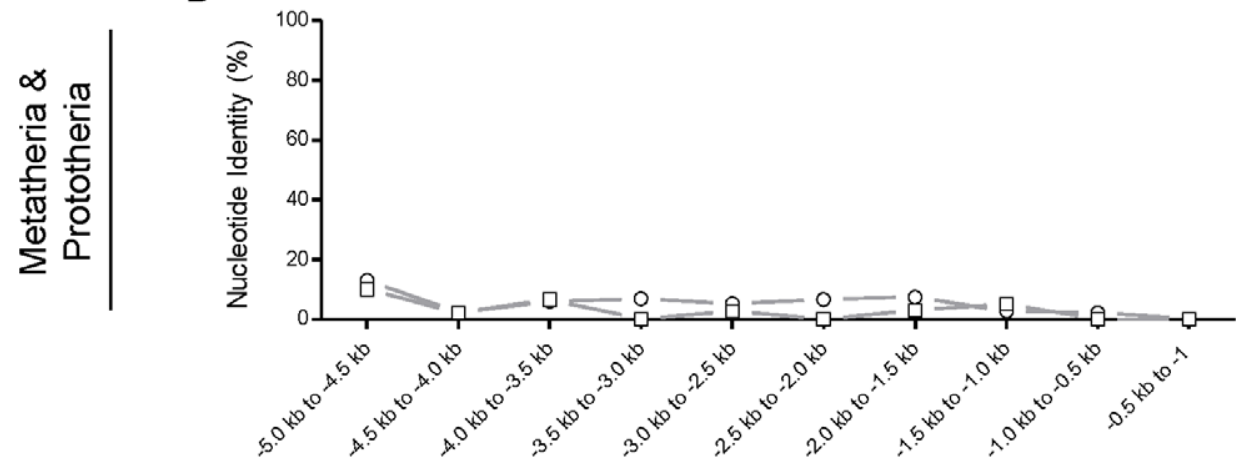

- B. taurus (Cow)

․ S. scrofa (Wild boar)

$\diamond$ C. lupus familiaris (Dog)

$\triangle$ A. melanoleuca (Giant panda)

$\nabla \quad$ E. caballus (Horse)

- O. cuniculus (European rabbit)
- M. domestica (Opossum)

○ O. anatinus (Platypus)

Figure 3. Conservation of the region upstream of $B m p 3$ between $M$. musculus and RefSeq mammal genomes. Pairwise alignments were performed between the Bmp3 upstream regions of $M$. musculus and all seventeen available RefSeq mammalian genomes (15 eutherian, 2 noneutherian) using BLAST 2.2.26+. Comparing sequential $500 \mathrm{nt}$ sections from M. musculus against the full-length $5 \mathrm{~kb}$ region upstream of $B m p 3$ from the other species allowed for the possibility of genomic insertions or deletions. Findings are separated into taxonomic classification for clarity (A-D). Actual numbers for these analyses are listed in Table S3. Conservation with $R$. norvegicus, $H$. sapiens, and B. taurus are based upon the primary assembly.

doi:10.1371/journal.pone.0057840.g003

Bmp3 upstream ECR varies (Table S4), prompting us to determine the minimal ECR that is conserved across all eutherian mammals in the RefSeq cohort. To do so, the full-length ECRs from each species were aligned using MUSCLE in MEGA5 [64], identifying a minimal $B m p 3$ upstream ECR (minECR) with a consensus length of $297 \mathrm{nt}$ (Figure 5). The mean identity to the consensus minECR is $90 \%$ (range: $79 \%-97 \%$, median: $92 \%$ ) (Table 1); the individual nucleotide conservation is shown in Figure S2.
The relative genomic location of the $B m p 3$ upstream minECR varies from species to species, but is quite consistent among closely-related species (Table 1). We found the $5^{\prime}$-end of the Bmp3 upstream minECR to be as close as position -741 in E. caballus and as distant as position -2772 in C. jacchus (Table 1). To determine if the minECR retains the repressive activity of the fulllength CoreECR, we placed both sequences upstream of the Thymidine Kinase minimal promoter. This revealed that the both were capable of repressing the Thymidine Kinase minimal 
A
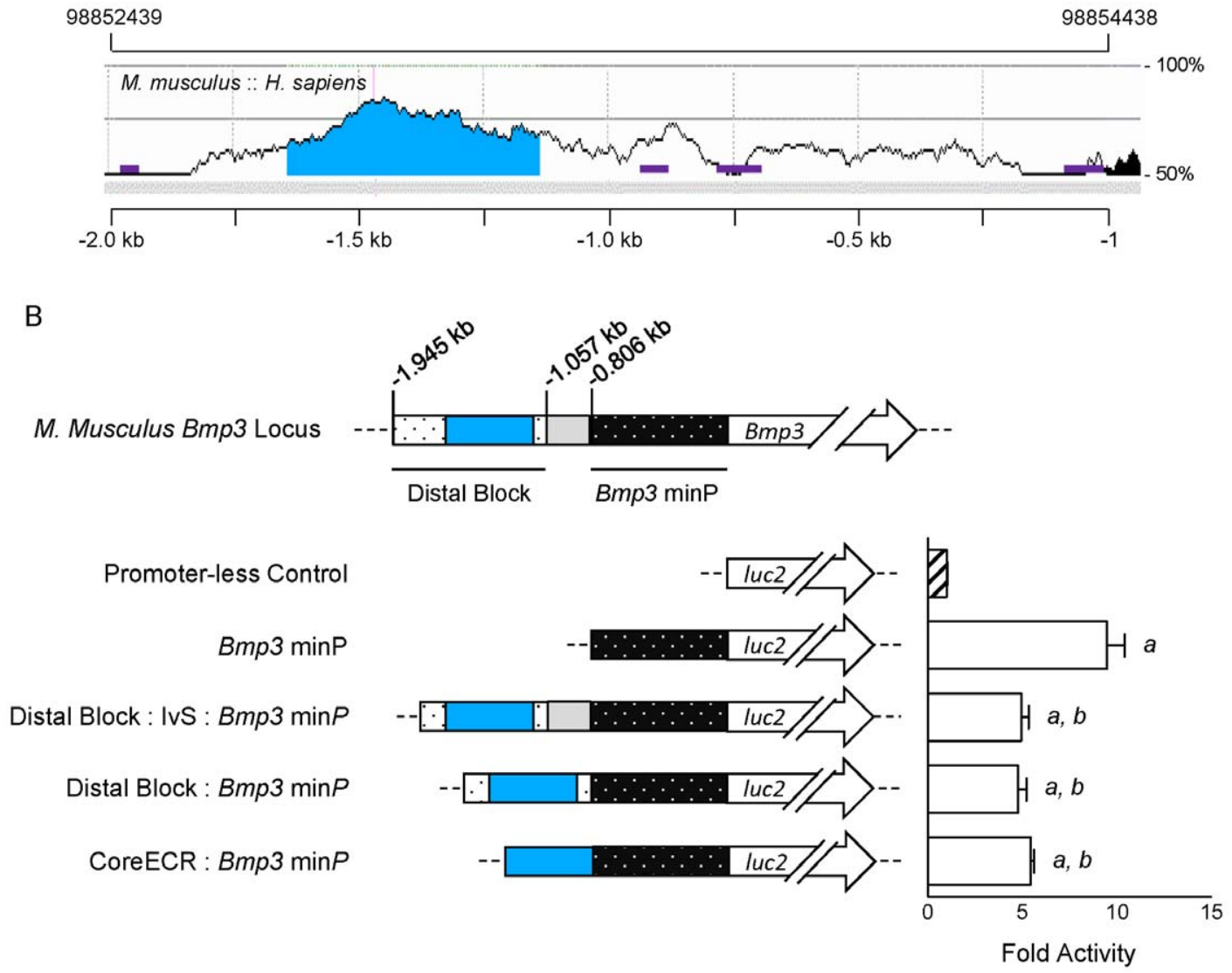

Figure 4. Identification of a repressive CoreECR upstream of Bmp3 between $\boldsymbol{M}$. musculus and $\boldsymbol{H}$. sapiens. A: Image adapted from ECR Browser aligning the regions upstream of the $B m p 3$ transcription start site between $M$. musculus and $H$. sapiens. Threshold was set to $\geq 77 \%$ identity across sliding $350 \mathrm{nt}$ window. This revealed a CoreECR upstream of the annotated transcription start site of Bmp3 in both species (blue region $-M$. musculus: 98852797-98853301 on NC_000071.6; H. sapiens: 81949936-81950336 on NC_000004.11). Genomic coordinates refer to location on M. musculus NC_000071.6. Gray bar indicates aligned segment. Purple boxes indicate repeat elements in M. musculus. Black region indicates $5^{\prime}$-end of Bmp3 exon 1 in M. musculus. B: Regulation of firefly luciferase activity driven by fragments from the region upstream of $M$. musculus Bmp3 in HEK293T cells. $\min \mathrm{P}=$ minimal promoter; IVS = intervening sequence between distal and proximal conserved blocks. Data are mean \pm SEM normalized to promoter-less control. $p<0.05$ as determined by One-way ANOVA with post hoc Newman-Keuls correction is indicated by " $a$ " versus promoter-less control and by " $\mathrm{b}$ " versus $-0.800 \mathrm{~kb}$ fragment.

doi:10.1371/journal.pone.0057840.g004

promoter, but the activity of the longer CoreECR was slightly stronger than the minECR (Figure 5B).

We analyzed the consensus minECR sequence using CENSOR [69] to identify potential DNA repeat elements, which revealed a reverse-orientation, partial match to the Short Interspersed Element (SINE) MIRb (Figure S2). However, inspection of the minECR from each individual species shows that this partial repeat is predominantly found in primates, and it resides in one of the more poorly aligned regions of the consensus minECR- the mean identity to this $57 \mathrm{nt}$ region is $88 \%$, and removing it from the consensus minECR increases the overall identity in 11/16 species, raising the mean identity to $91 \%$. For this reason, and the small size of this partial repeat region relative to the full minECR, we do not credit the high conservation of the $B m p 3$ upstream minECR across eutherian mammals to a conserved retrotransposon.

Additionally, we confirmed that the $B m p 3$ minECR resides in a non-coding region of the genome by performing BLASTN alignment of the consensus Bmp3 upstream minECR against the database of GenBank/EMBL/DDBJ expressed sequence tags
(ESTs), which comprise $>73,580,051$ sequences. This failed to identify any EST with significant alignment to the consensus Bmp3 upstream minECR- the highest identity was $85 \%$ over 59 nt (JWL, data not shown). For comparison, exon 1 of M. musculus Bmp3 readily aligned to multiple ESTs from $M$. musculus and other species (JWL, data not shown).

Identification of the consensus Bmp3 upstream minECR in all available eutherian mammal genomes

Our findings suggest that the $B m p 3$ upstream ECR is a cisregulatory element unique to eutherian mammals. However, since the number of RefSeq genomes is fairly small at present, we extended our investigation to species for which a RefSeq genome is not available. We used BLASTN to align the consensus Bmp3 upstream minECR to the whole-genome shotgun sequence database, which comprises 191 animal species. This revealed significant conservation of the $B m p 3$ upstream minECR in thirtyone of thirty-nine eutherian mammals that were not represented by the RefSeq cohort (Table S5); notably, at the present stage of their assembly, there is no identifiable $B m p 3$ gene in any of the 


\author{
1 tTtCCAgggc CTattGaAt TTGCaaTTag gTcTtcattA tTAATcACAA \\ 51 aagctattat gttctttatg CCaagcaaag aTCAcaCACa tgatctcatt \\ 101 taatcctcac aaCcActct gaagTatgtg ttatTaTcTg cAGaacaGTT \\ 151 cccggggtGg CtaagGGcaa aatcCaaACc gcagtgggtt gagaAgtGac \\ 201 tgagtGgGGa GaatgtaGtC TGcatTtggG AtaaAtTgG GAtagaAagg \\ 251 GAtTgAGccc ctActaAAgg ctagtcagtg nGCCagtCac tttAgca
}

B

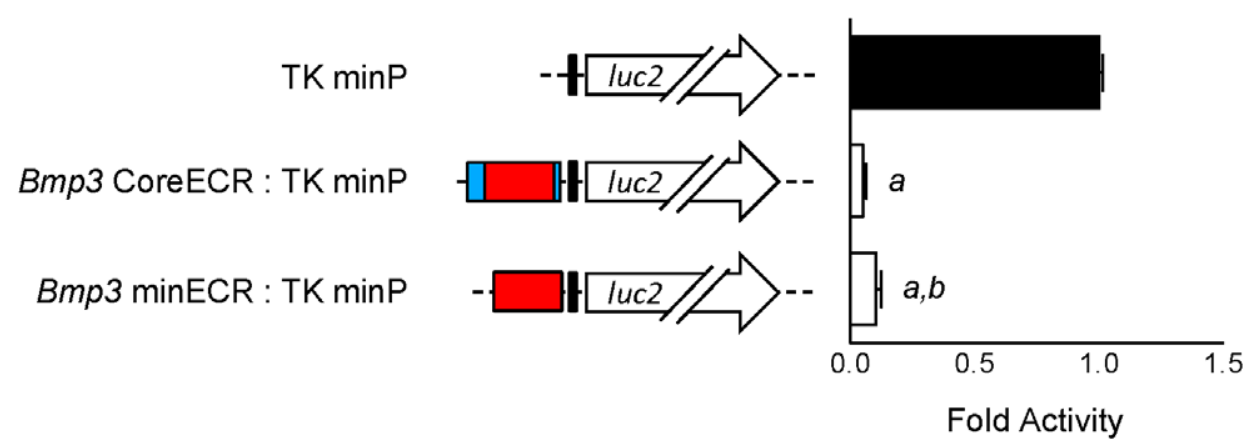

Figure 5. Examination of the minimal $B m p 3$ upstream ECR (minECR) present in all RefSeq mammals. BLASTN (Version 2.2.26+) was used to align the $M$. musculus: $H$. sapiens CoreECR sequence to all sixteen available eutherian mammal RefSeq genomes in order to identify the $B m p 3$ upstream ECR shared with M. musculus. Each full-length ECR was then aligned using MUSCLE in MEGA5 [64], identifying a minimal Bmp3 upstream ECR (minECR) with a consensus length of $297 \mathrm{nt}$. The mean identity to the consensus minECR is $90 \%$ (range: $79 \%-97 \%$, median: $92 \%$ ); the individual nucleotide conservation is shown in Figure S2. Uppercase letters in the consensus sequence indicate $100 \%$ conservation, while lowercase letters indicate the majority nucleotide; " $n$ " indicates no consensus nucleotide could be determined. B: Regulation of Thymidine Kinase minimal promoter (TK minP) by fragments from the region upstream of M. musculus Bmp3 in HEK293T cells. Data are mean \pm SEM normalized to promoter-less control. $p<0.05$ as determined by One-way ANOVA with post hoc Newman-Keuls correction is indicated by " $a$ " versus promoter-less control and by "b" versus CoreECR fragment.

doi:10.1371/journal.pone.0057840.g005

eight eutherian mammals in which the $B m p 3$ upstream ECR was not identified. Moreover, although the $B m p 3$ gene has been found in at least sixteen non-eutherian animals in this databse, the $B m p 3$ upstream minECR was not found in any of the 136 non-eutherian animal genomes analyzed (JWL, data not shown).

\section{Identification of transcription factor binding sites in the consensus Bmp3 upstream ECR}

Next, we turned our focus to examining the potential cisregulatory role(s) played by the $B m p 3$ upstream ECR. To do so, we first used rVista 2.0 to identify the transcription factor binding sites (TFBSs) in the consensus Bmp3 minECR sequence (Table S6). This yielded a list of fifty-one distinct binding sites, the majority of which are estimated to be high affinity by TRAP analysis and are pathway-specific transcription factors (eg, C/EBP, Ikaros, AP1).

Finally, we sought to validate the evolutionary conservation of the Bmp3 upstream minECR as a cluster of cis-regulatory elements by determining the degree to which conserved cis-regulatory elements exist outside of the minECR. To do so, we aligned the regions upstream of $M$. musculus and $H$. sapiens, then identified conserved transcription factor binding sites using rVista 2.0 (Table S7). This revealed that, with the exception of one (STAT3), all of the transcription factor binding sites within $2 \mathrm{~kb}$ upstream of $B m p 3$ in M. musculus that are conserved with $H$. sapiens reside within the M. musculus: $H$. sapiens CoreECR (Figure 6 and Table S7). Moreover, sixteen of the twenty $(80 \%)$ transcription factor binding sites conserved between $M$. musculus and $H$. sapiens upstream of Bmp3 reside within the limits of the minECR, and all but one
(USF) are present in the consensus minECR sequence (Figure 6 and Table S7). For secondary confirmation of this finding, we aligned the regions upstream of $B m p 3$ from M. musculus, $R$. norvegicus, C. griseus, and Guinea pig (C. porcellus). This revealed that all of the TFBSs within $3.8 \mathrm{~kb}$ upstream of $B \mathrm{mp} 3$ that are conserved across the RefSeq rodent genomes (9/9) reside within the limits of the minECR and are present in the consensus minECR sequence (JWL, data not shown). These findings indicate that the consensus minECR could serve as a valid predictor of highly conserved cis-regulatory elements that govern $B m p 3$ expression.

\section{Discussion}

In the present study, we took a comparative genomics approach to identify potential cis-regulatory elements controling Bmp3 expression in $M$. musculus. Functional characterization of various genomic fragments revealed the $0.8 \mathrm{~kb}$ region proximal to the annotated M. musculus Bmp3 transcription start site to be the minimal promoter in HEK293T cells, UMR-106 osteosarcoma cells, and primary calvarial osteoblasts. This region corresponds to a highly conserved block $(\geq 80 \%$ identity across a sliding $100 \mathrm{nt}$ window) that is shared between $M$. musculus and $R$. norvegicus and contains binding sites for a number of both general and pathwayspecific transcription factors. We analyzed the $5 \mathrm{~kb}$ upstream region and exon 1 of Bmp3 from M. musculus using Neural Network Promoter Scan [71] to identify potential transcription starts sites (TSSs) within the minimal promoter. This revealed two TSSs 
Table 1. Conservation of the consensus minimal Bmp3 upstream Evolutionary Conserved Region (minECR) in RefSeq animal genomes.

\begin{tabular}{|c|c|c|c|c|c|c|}
\hline & & \multirow[t]{2}{*}{ Accession Number } & \multicolumn{2}{|l|}{$\min E C R$} & \multicolumn{2}{|c|}{ Distance from $B m p 3$ (nt) } \\
\hline & & & Location & $\%$ Identity & $\mathbf{5}^{\prime}$ & $\mathbf{3}^{\prime}$ \\
\hline \multirow[t]{5}{*}{ Rodentia } & M. musculus & NC_000071.6 & 95552942.95553250 & $79 \%$ & -1497 & -1189 \\
\hline & R. norvegicus & NC_005113.3 & 12332541.12332253, complement & $82 \%$ & -1511 & -1223 \\
\hline & & AC_000082.1 & 10827337.10827049, complement & $82 \%$ & -1511 & -1223 \\
\hline & C. porcellus & NT_176414.1 & 5088909.5089198 & $80 \%$ & -1789 & -1500 \\
\hline & C. griseus & NW_003616697.1 & 55307.54999, complement & $82 \%$ & -1611 & -1303 \\
\hline \multirow[t]{7}{*}{ Primates } & H. sapiens & NC_000004.11 & 81950009.81950305 & $97 \%$ & -2110 & -1814 \\
\hline & & AC_000136.1 & 77693899.77694195 & $97 \%$ & -2111 & -1815 \\
\hline & P. troglodytes & NC_006471.3 & 49002602.49002306, complement & $97 \%$ & -2101 & -1805 \\
\hline & P. abelii & NC_012595.1 & 84489849.84490145 & $97 \%$ & -2120 & -1824 \\
\hline & M. mulatta & NC_007862.1 & 48535985.48535688, complement & $96 \%$ & -2137 & -1840 \\
\hline & N. leucogenys & NW_003501411.1 & 13030680.13030976 & $96 \%$ & -2109 & -1813 \\
\hline & C. jacchus & NC_013898.1 & 113372870.113372574, complement & $96 \%$ & -2772 & -2476 \\
\hline \multirow[t]{7}{*}{ Other Orders } & B. taurus & AC_000163.1 & 97583173.97583469 & $93 \%$ & -2360 & -2064 \\
\hline & & NC_007304.5 & 99175053.99175349 & $93 \%$ & -2360 & -2064 \\
\hline & S. scrofa & NC_010450.3 & 146200934.146200644, complement & $78 \%$ & -1914 & -1624 \\
\hline & C. lupus familiaris & NC_006614.2 & 8169902.8170198 & $87 \%$ & -2135 & -1839 \\
\hline & A. melanoleuca & NW_003217292.1 & 2528248.2527953, complement & $92 \%$ & -2167 & -1872 \\
\hline & E. caballus & NC_009146.2 & 55766669.55766369, complement & $90 \%$ & -741 & -441 \\
\hline & O. cuniculus & NC_013683.1 & 69240613.69240317, complement & $86 \%$ & -2347 & -2051 \\
\hline
\end{tabular}

Species are separated by taxonomic order. Distance from $\mathrm{Bmp} 3$ is calculated from the annotated transcription start site. nt: nucleotide.

doi:10.1371/journal.pone.0057840.t001

$(-0.108 \mathrm{~kb}$ and $-0.452 \mathrm{~kb})$ upstream of exon 1 . These two TSSs, in addition to the annotated TSS, are contained in our luciferase reporter plasmids. Thus, we are unable to determine which TSS is used in each cell type; however, the $0.8 \mathrm{~kb}$ fragment consistently led to induction of luciferase expression in each of our assays whereas shorter fragments did not, leading us to conclude that we have identified the minimal region necessary to drive $B m p 3$ expression. Our findings are consistent with a previous report that attained promoter activity using the $2 \mathrm{~kb}$ region upstream of $B m p 3$ in $R$. norvegicus [25].

We were surprised to find that the $B m p 3$ minimal promoter is poorly conserved between $M$. musculus and $H$. sapiens, although this finding is consistent with a previous report comparing the promoter for $B m p 3 b$ (also known as GDF10) between M. musculus and $H$. sapiens [9]. While $B m p 3 b$ likely arose from duplication of the Bmp3 gene, or vice versa $[9,72]$, alignment of the $5 \mathrm{~kb}$ regions upstream of $B m p 3$ and $B m p 3 b$ in M. musculus failed to demonstrate any significant nucleotide identity (JWL, data not shown), suggesting that $B m p 3$ and $B m p 3 b$ have evolved unique mechanisms regulating their expression. This idea is supported by the fact that the spatio-temporal expression domains of $B m p 3$ and $B m p 3 b$ differ quite drastically [72].

After identifying the proximal block conserved between $M$. musculus and $R$. norvegicus as the minimal promoter, we turned our attention to the remaining highly conserved region $(-1.9 \mathrm{~kb}$ to $-0.8 \mathrm{~kb}$ upstream of $\mathrm{Bmp} 3$ ). Interestingly, appending this region to

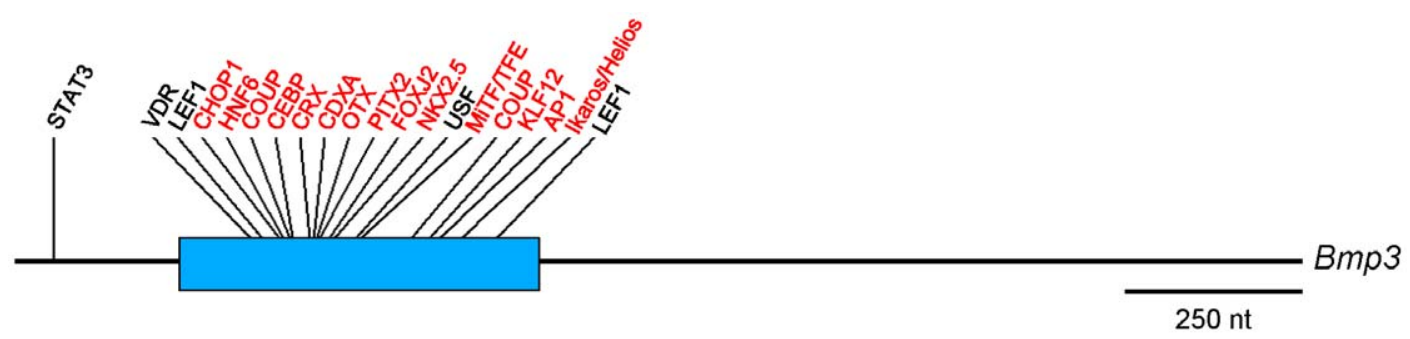

Figure 6. Transcription factor binding sites conserved between $M$. musculus and $H$. sapiens in the Bmp3 upstream region. Transcription factor binding sites in the $1.797 \mathrm{~kb}$ genomic fragment were identified using rVista 2.0 for M. musculus and $H$. sapiens. With the exception of one, all transcription factor binding sites within $2 \mathrm{~kb}$ upstream of Bmp3 that are conserved between $M$. musculus and $\mathrm{H}$. sapiens lie within the CoreECR. Transcription factor binding sites in red are also conserved with the consensus Bmp3 upstream minECR. Drawn to scale using the $M$. musculus $1.797 \mathrm{~kb}$ genomic fragment from Figure 1.

doi:10.1371/journal.pone.0057840.g006 
the minimal promoter repressed promoter activity. Fidelity of the genomic fragment in this reporter plasmid was confirmed by bidirectional sequencing and our finding was consistent in each repetition of our assay, leading us to conclude that this region is capable of repressing basal Bmp3 expression in each cell type tested. This is supported by the fact that, when we examined potential TFBSs in this region by rVista2.0 [66] analysis, we identified binding sites for a number of potential repressive transcription factors.

BLASTN alignment of the $5 \mathrm{~kb}$ regions between $M$. musculus and each of the other thirty-nine complete RefSeq genomes revealed that a portion of the distal block was conserved with every eutherian mammal $(15 / 15)$, but not in any non-eutherian species $(0 / 24)$ even though a $B m p 3$ or Bmp3-like locus has been annotated for at least thirteen non-eutherian species (eg, X. tropicalis, D. rerio, $G$. gallus, $O$. anatinus, $M$. domestica). We went on to narrow this conserved region to $297 \mathrm{nt}$ that is shared between all eutherian mammals in the RefSeq cohort, and then found this minimal ECR in thirty-one additional eutherian mammals represented in the whole-genome shotgun sequence (WGS) database (total of fortyseven eutherian mammals between RefSeq and WGS databases). As with the RefSeq database, we did not find the minimal ECR in any of the 136 non-eutherian animal genomes in the WGS database. Of note, genomic sequences are presently available from only four non-eutherian mammals (O. anatinus, $M$. domestica, $M$. eugenii and $S$. harissii). Thus, we conclude from our findings that the Bmp3 upstream ECR is a eutherian mammal-specific cis-element, but are aware that future studies are required to definitively show if this ECR is also found in metatherian or prototherian mammals.

The Bmp3 upstream minECR is a highly conserved genomic region near the minimal promoter that represses basal promoter activity. This arrangement is similar to the conserved, high GCcontent short-range repressive elements that have been described near the Bmp2 promoter [73] -though the Bmp3 upstream minECR bears no alignment to these regions and the GC content is only $45 \%$ (JWL, data not shown). To examine possible regulatory mechanisms, we examined the $B m p 3$ upstream minECR using rVista 2.0, which not only pinpoints consensus TFBSs using the TRANSFAC database but combines this information with sequence conservation analyses of the surrounding $20 \mathrm{nt}$ to identify the most biologically relevant TFBSs [66], and TRAP analysis, which predicts transcription factor binding affinity to each site $[67,68]$. This revealed that sixteen of the twenty $(80 \%)$ transcription factor binding sites conserved between M. musculus and $H$. sapiens upstream of $B m p 3$ reside within the limits of the minECR, and the majority of these are predicted to be high-affinity binding sites. Moreover, even among the more closely-related rodents $M$. musculus, $R$. norvegicus, $C$. griseus, and $C$. porcellus we found that all of the TFBSs within $3.8 \mathrm{~kb}$ upstream of Bmp3 (9/9) reside within the limits of the minECR (JWL, data not shown).

Our identification of a highly conserved block of potential cisregulatory elements upstream of $B m p 3$ in mammals provides a foundation for future studies examining modulation of $B m p 3$ expression. In support of this, the TFBSs that we identified are highly consistent with what has previously been reported on the regulation of Bmp3. For instance, binding sites for HNF1, VDR, AP1, and NF-kappaB, all of which have been shown to regulate Bmp3 expression [25,52,55,57,60], are present in the Bmp3 upstream minECR.

Of particular interest to us is the role of $B m p 3$ in regulation of bone formation. Similar to many osteogenic BMP ligands, Bmp3 is expressed in osteoblasts $[17,45,46,53,54]$. However, while canonical BMP signaling is required for bone formation (reviewed in
[74]), Bmp3 knockout mice have high bone mass [15] and overexpression of BMP3 leads to spontaneous rib fractures in mice [22], indicating that $\mathrm{BMP} 3$ is a negative regulator of osteogenesis. As such, the identification of a highly-conserved repressive element near the $B m p 3$ promoter could determine mechanisms to reduce $B m p 3$ expression in diseases of low bone mass such as osteopenia and osteoporosis.

\section{Concluding Remarks}

We identified the minimal Bmp3 promoter from M. musculus and determined that this region is highly conserved with $R$. norvegicus. We also found that a highly conserved upstream region abutting the minimal promoter is able to repress the minimal promoter. A portion of this region is conserved among all available eutherian mammal genomes $(47 / 47)$, but not in any non-eutherian animal (0/136). We also identified multiple conserved transcription factor binding sites in the $B m p 3$ upstream ECR. Collectively, these findings suggest that the high level of conservation of the $B m p 3$ upstream ECR may preserve common cis-regulatory elements that govern Bmp3 expression across eutherian mammals.

\section{Supporting Information}

Figure S1 Bmp3 expression analysis. RT-PCR for $B m p 3$ in HEK293T cells $(\mathbf{A})$, UMR-106 cells $(\mathbf{B})$, primary mouse calvarial osteoblasts(cOBs, C), and C2C12 cells 9D) compared to Hprt housekeeping control. Newborn mouse hind limb cDNA was used as a positive control in all experiments (only shown in $\mathbf{A}$ ). Intervening lanes from a single gel removed in A (indicated by white bar).

(TIFF)

Figure S2 Determination and individual nucleotide conservation of the minimal Bmp3 upstream EGR (minEGR) present in all RefSeq mammals. BLASTN (Version 2.2.26+) was used to align the M. musculus: H. sapiens CoreECR sequence to all sixteen available eutherian mammal RefSeq genomes in order to identify the $B m p 3$ upstream ECR shared with $M$. musculus. Each full-length ECR was then aligned using MUSCLE in MEGA5 [64], identifying a minimal Bmp3 upstream ECR (minECR) with a consensus length of $297 \mathrm{nt}$ (319 nt as shown when including insertions found in some species). The consensus sequence was determined using Los Alamos National Laboratory's Simple Consensus Maker. Uppercase letters in the consensus sequence indicate $100 \%$ conservation, while lowercase letters indicate the majority nucleotide. " $n$ " indicates no consensus nucleotide could be determined and "." indicates a gap. For each individual species, a dash indicates a match to the consensus, while "A, T, $\mathrm{C}$, or $\mathrm{G}$ " indicates a mismatch to the consensus. The mean identity to the consensus minECR is $90 \%$ (range: 79\%-97\%, median: 92\%). A partial match to the SINE2type repeat MIRb, found primarily in primates, is denoted in red. (TIFF)

Table S1 Primers used for firefly luciferase reporter plasmid construction.

(XLSX)

Table S2 Transcription factor binding sites upstream of $M$. musculus Bmp3.

(XLSX)

Table S3 Nucleotide alignments of the Bmp3 $5 \mathrm{~kb}$ upstream regions between $M$. musculus and other mammals.

(XLS) 
Table S4 Conservation analysis of the $B m p 3$ upstream Evolutionary Conserved Region (ECR) in RefSeq animal genomes. (XLS)

Table S5 Conservation analysis of the minimal $B m p 3$ upstream Evolutionary Conserved Region (minECR) in all available animal genomes.

\section{(XLS)}

Table S6 Transcription factor binding sites in the consensus minimal Bmp3 upstream ECR and conservation in Rodents/ Primates.

\section{(XLSX)}

\section{References}

1. Newfeld SJ, Wisotzkey RG, Kumar S (1999) Molecular evolution of a developmental pathway: phylogenetic analyses of transforming growth factorbeta family ligands, receptors and Smad signal transducers. Genetics 152: 783795.

2. Holstein TW, Watanabe H, Ozbek S (2011) Signaling pathways and axis formation in the lower metazoa. Current topics in developmental biology 97: $137-177$.

3. Urist MR (1965) Bone: formation by autoinduction. Science 150: 893-899.

4. Zhao GQ (2003) Consequences of knocking out BMP signaling in the mouse. Genesis 35: 43-56.

5. Kishigami S, Mishina Y (2005) BMP signaling and early embryonic patterning. Cytokine Growth Factor Rev 16: 265-278.

6. Murakami K, Mathew R, Huang J, Farahani R, Peng H, et al. (2010) Smurfl ubiquitin ligase causes downregulation of BMP receptors and is induced in monocrotaline and hypoxia models of pulmonary arterial hypertension. Experimental biology and medicine 235: 805-813.

7. Inoue Y, Imamura T (2008) Regulation of TGF-beta family signaling by E3 ubiquitin ligases. Cancer science 99: 2107-2112.

8. Lapraz F, Rottinger E, Duboc V, Range R, Duloquin L, et al. (2006) RTK and TGF-beta signaling pathways genes in the sea urchin genome. Developmental biology 300: 132-152.

9. Katoh Y, Katoh M (2006) Comparative integromics on BMP/GDF family. International journal of molecular medicine 17: 951-955.

10. Lowery JW, de Caestecker MP (2010) BMP signaling in vascular development and disease. Cytokine Growth Factor Rev 21: 287-298.

11. Takao M, Hino J, Takeshita N, Konno Y, Nishizawa T, et al. (1996) Identification of rat bone morphogenetic protein-3b (BMP-3b), a new member of BMP-3. Biochemical and biophysical research communications 219: 656-662.

12. Reddi AH, Muthukumaran N, Ma S, Carrington JL, Luyten FP, et al. (1989) Initiation of bone development by osteogenin and promotion by growth factors. Connective tissue research 20: 303-312.

13. Wozney JM, Rosen V, Celeste AJ, Mitsock LM, Whitters MJ, et al. (1988) Novel regulators of bone formation: molecular clones and activities. Science 242: 1528-1534.

14. Chen G, Deng C, Li YP (2012) TGF-beta and BMP signaling in osteoblast differentiation and bone formation. International journal of biological sciences 8: 272-288.

15. Daluiski A, Engstrand T, Bahamonde ME, Gamer LW, Agius E, et al. (2001) Bone morphogenetic protein- 3 is a negative regulator of bone density. Nature genetics 27: 84-88.

16. Bahamonde ME, Lyons KM (2001) BMP3: to be or not to be a BMP. The Journal of bone and joint surgery American volume 83-A Suppl 1: S56-62.

17. Kokabu S, Gamer L, Cox K, Lowery J, Tsuji K, et al. (2012) BMP3 suppresses osteoblast differentiation of bone marrow stromal cells via interaction with Acvr2b. Molecular endocrinology 26: 87-94.

18. Luu HH, Song WX, Luo X, Manning D, Luo J, et al. (2007) Distinct roles of bone morphogenetic proteins in osteogenic differentiation of mesenchymal stem cells. Journal of orthopaedic research : official publication of the Orthopaedic Research Society 25: 665-677.

19. Gamer LW, Ho V, Cox K, Rosen V (2008) Expression and function of BMP3 during chick limb development. Developmental dynamics : an official publication of the American Association of Anatomists 237: 1691-1698.

20. Gamer LW, Nove J, Levin M, Rosen V (2005) BMP-3 is a novel inhibitor of both activin and BMP-4 signaling in Xenopus embryos. Developmental biology 285: $156-168$.

21. Hino J, Nishimatsu S, Nagai T, Matsuo H, Kangawa K, et al. (2003) Coordination of BMP-3b and cerberus is required for head formation of Xenopus embryos. Developmental biology 260: 138-157.

22. Gamer LW, Cox K, Carlo JM, Rosen V (2009) Overexpression of BMP3 in the developing skeleton alters endochondral bone formation resulting in spontaneous rib fractures. Developmental dynamics : an official publication of the American Association of Anatomists 238: 2374-2381.
Table S7 Transcription factor binding sites conserved between M. musculus and H. sapiens in the region upstream of Bmp3. (XLS)

\section{Acknowledgments}

We would like to thank Dr. Laura Gamer for critical reading of the manuscript.

\section{Author Contributions}

Conceived and designed the experiments: JWL AWL SK VR. Performed the experiments: JWL AWL SK. Analyzed the data: JWL AWL SK VR. Wrote the paper: JWL VR.

23. Allendorph GP, Vale WW, Choe S (2006) Structure of the ternary signaling complex of a TGF-beta superfamily member. Proc Natl Acad Sci USA 103: 7643-7648.

24. Stewart A, Guan H, Yang K (2010) BMP-3 promotes mesenchymal stem cell proliferation through the TGF-beta/activin signaling pathway. Journal of cellular physiology 223: 658-666.

25. Bonner C, Farrelly AM, Concannon CG, Dussmann H, Baquie M, et al. (2011) Bone morphogenetic protein 3 controls insulin gene expression and is downregulated in INS-1 cells inducibly expressing a hepatocyte nuclear factor 1Amaturity-onset diabetes of the young mutation. The Journal of biological chemistry 286: 25719-25728.

26. Dwivedi PP, Anderson PJ, Powell BC (2012) Development of an efficient, nonviral transfection method for studying gene function and bone growth in human primary cranial suture mesenchymal cells reveals that the cells respond to BMP2 and BMP3. BMC biotechnology 12: 45.

27. Yamamoto M, Beppu H, Takaoka K, Meno C, Li E, et al. (2009) Antagonism between Smad1 and Smad2 signaling determines the site of distal visceral endoderm formation in the mouse embryo. The Journal of cell biology 184: 323-334.

28. Goumans MJ, Lebrin F, Valdimarsdottir G (2003) Controlling the angiogenic switch: a balance between two distinct TGF-b receptor signaling pathways. Trends in cardiovascular medicine 13: 301-307.

29. Nguyen TQ, Goldschmeding R (2008) Bone morphogenetic protein-7 and connective tissue growth factor: novel targets for treatment of renal fibrosis? Pharmaceutical research 25: 2416-2426.

30. Izumi N, Mizuguchi S, Inagaki Y, Saika S, Kawada N, et al. (2006) BMP-7 opposes TGF-betal-mediated collagen induction in mouse pulmonary myofibroblasts through Id2. Am J Physiol Lung Cell Mol Physiol 290: L120-126.

31. Wordinger RJ, Fleenor DL, Hellberg PE, Pang IH, Tovar TO, et al. (2007) Effects of TGF-beta2, BMP-4, and gremlin in the trabecular meshwork: implications for glaucoma. Investigative ophthalmology \& visual science 48: 1191-1200.

32. Zode GS, Clark AF, Wordinger RJ (2009) Bone morphogenetic protein 4 inhibits TGF-beta2 stimulation of extracellular matrix proteins in optic nerve head cells: role of gremlin in ECM modulation. Glia 57: 755-766.

33. Davies RJ, Morrell NW (2008) Molecular mechanisms of pulmonary arterial hypertension: role of mutations in the bone morphogenetic protein type II receptor. Chest 134: 1271-1277.

34. Koncarevic A, Cornwall-Brady M, Pullen A, Davies M, Sako D, et al. (2010) A soluble activin receptor type IIb prevents the effects of androgen deprivation on body composition and bone health. Endocrinology 151: 4289-4300.

35. Cadena SM, Tomkinson KN, Monnell TE, Spaits MS, Kumar R, et al. (2010) Administration of a soluble activin type IIB receptor promotes skeletal muscle growth independent of fiber type. Journal of applied physiology 109: 635-642.

36. Lotinun S, Pearsall RS, Davies MV, Marvell TH, Monnell TE, et al. (2010) A soluble activin receptor Type IIA fusion protein (ACE-011) increases bone mass via a dual anabolic-antiresorptive effect in Cynomolgus monkeys. Bone 46: 1082-1088.

37. Fajardo RJ, Manoharan RK, Pearsall RS, Davies MV, Marvell T, et al. (2010) Treatment with a soluble receptor for activin improves bone mass and structure in the axial and appendicular skeleton of female cynomolgus macaques (Macaca fascicularis). Bone 46: 64-71.

38. Edwards JR, Nyman JS, Lwin ST, Moore MM, Esparza J, et al. (2010) Inhibition of TGF-beta signaling by 1D11 antibody treatment increases bone mass and quality in vivo. Journal of bone and mineral research : the official journal of the American Society for Bone and Mineral Research 25: 2419-2426.

39. Ku GM, Kim H, Vaughn IW, Hangauer MJ, Oh CM, et al. (2012) Research Resource: RNA-Seq Reveals Unique Features of the Pancreatic beta-Cell Transcriptome. Molecular endocrinology.

40. Cho TJ, Gerstenfeld LC, Einhorn TA (2002) Differential temporal expression of members of the transforming growth factor beta superfamily during murine fracture healing. Journal of bone and mineral research : the official journal of the American Society for Bone and Mineral Research 17: 513-520. 
41. Gil H, Lozano JJ, Alvarez-Garcia O, Secades-Vazquez P, Rodriguez-Suarez J, et al. (2008) Differential gene expression induced by growth hormone treatment in the uremic rat growth plate. Growth hormone \& IGF research : official journal of the Growth Hormone Research Society and the International IGF Research Society 18: 353-359.

42. Haque T, Hamade F, Alam N, Kotsiopriftis M, Lauzier D, et al. (2008) Characterizing the BMP pathway in a wild type mouse model of distraction osteogenesis. Bone 42: 1144-1153.

43. Hardy G, Kramer B (2000) Spatial and temporal localisation of bone morphogenetic protein-3 (osteogenin) in the developing rat submandibular gland. SADJ : journal of the South African Dental Association = tydskrif van die Suid-Afrikaanse Tandheelkundige Vereniging 55: 136-141.

44. Jaatinen R, Rosen V, Tuuri T, Ritvos O (1996) Identification of ovarian granulosa cells as a novel site of expression for bone morphogenetic protein-3 (BMP-3/osteogenin) and regulation of BMP-3 messenger ribonucleic acids by chorionic gonadotropin in cultured human granulosa-luteal cells. The Journal of clinical endocrinology and metabolism 81: 3877-3882.

45. Kemoun P, Laurencin-Dalicieux S, Rue J, Vaysse F, Romeas A, et al. (2007) Localization of STRO-1, BMP-2/-3/-7, BMP receptors and phosphorylated Smad-1 during the formation of mouse periodontium. Tissue \& cell 39: 257266.

46. Kloen P, Di Paola M, Borens O, Richmond J, Perino G, et al. (2003) BMP signaling components are expressed in human fracture callus. Bone 33: 362-371.

47. Nie X (2005) Apoptosis, proliferation and gene expression patterns in mouse developing tongue. Anatomy and embryology 210: 125-132.

48. Nie XG (2005) [Differential expression of Bmp2, Bmp4 and Bmp3 in embryonic development of mouse anterior and posterior palate]. Chinese medical journal 118: 1710-1716.

49. Takahashi H, Ikeda T (1996) Transcripts for two members of the transforming growth factor-beta superfamily BMP-3 and BMP-7 are expressed in developing rat embryos. Developmental dynamics : an official publication of the American Association of Anatomists 207: 439-449.

50. Thomadakis G, Ramoshebi LN, Crooks J, Rueger DC, Ripamonti U (1999) Immunolocalization of Bone Morphogenetic Protein-2 and -3 and Osteogenic Protein-1 during murine tooth root morphogenesis and in other craniofacial structures. European journal of oral sciences 107: 368-377.

51. Yamashiro T, Tummers M, Thesleff I (2003) Expression of bone morphogenetic proteins and Msx genes during root formation. Journal of dental research 82: $172-176$.

52. Yao S, Prpic V, Pan F, Wise GE (2010) TNF-alpha upregulates expression of BMP-2 and BMP-3 genes in the rat dental follicle-implications for tooth eruption. Connective tissue research 51: 59-66.

53. Zheng L, Yamashiro T, Fukunaga T, Balam TA, Takano-Yamamoto T (2005) Bone morphogenetic protein 3 expression pattern in rat condylar cartilage, femoral cartilage and mandibular fracture callus. European journal of oral sciences 113: 318-325.

54. Zoricic S, Maric I, Bobinac D, Vukicevic S (2003) Expression of bone morphogenetic proteins and cartilage-derived morphogenetic proteins during osteophyte formation in humans. Journal of anatomy 202: 269-277.

55. Faucheux C, Bareille R, Amedee J, Triffitt JT (1999) Effect of 1,25(OH)2D3 on bone morphogenetic protein-3 mRNA expression. Journal of cellular biochemistry 73: 11-19.

56. Johnson KJ, Robbins AK, Wang Y, McCahan SM, Chacko JK, et al. (2010) Insulin-like 3 exposure of the fetal rat gubernaculum modulates expression of genes involved in neural pathways. Biology of reproduction 83: 774-782.
57. Martinovic S, Borovecki F, Miljavac V, Kisic V, Maticic D, et al. (2006) Requirement of a bone morphogenetic protein for the maintenance and stimulation of osteoblast differentiation. Archives of histology and cytology 69: 23-36.

58. Nacamuli RP, Fong KD, Lenton KA, Song HM, Fang TD, et al. (2005) Expression and possible mechanisms of regulation of BMP3 in rat cranial sutures. Plastic and reconstructive surgery 116: 1353-1362.

59. Onyia JE, Helvering LM, Gelbert L, Wei T, Huang S, et al. (2005) Molecular profile of catabolic versus anabolic treatment regimens of parathyroid hormone (PTH) in rat bone: an analysis by DNA microarray. Journal of cellular biochemistry 95: 403-418.

60. Qin L, Qiu P, Wang L, Li X, Swarthout JT, et al. (2003) Gene expression profiles and transcription factors involved in parathyroid hormone signaling in osteoblasts revealed by microarray and bioinformatics. The Journal of biological chemistry 278: 19723-19731.

61. Owen TA, Pan LC (2008) Isolation and culture of rodent osteoprogenitor cells. Methods in molecular biology 455: 3-18.

62. Zhang Z, Schwartz S, Wagner L, Miller W (2000) A greedy algorithm for aligning DNA sequences. Journal of computational biology : a journal of computational molecular cell biology 7: 203-214.

63. Ovcharenko I, Nobrega MA, Loots GG, Stubbs L (2004) ECR Browser: a tool for visualizing and accessing data from comparisons of multiple vertebrate genomes. Nucleic acids research 32: W280-286.

64. Tamura K, Peterson D, Peterson N, Stecher G, Nei M, et al. (2011) MEGA5 Molecular Evolutionary Genetics Analysis using Maximum Likelihood, Evolutionary Distance, and Maximum Parsimony Methods. Molecular biology and evolution.

65. Ovcharenko I, Loots GG, Hardison RC, Miller W, Stubbs L (2004) zPicture: dynamic alignment and visualization tool for analyzing conservation profiles. Genome research 14: 472-477.

66. Loots GG, Ovcharenko I (2004) rVISTA 2.0: evolutionary analysis of transcription factor binding sites. Nucleic acids research 32: W217-221.

67. Roider HG, Kanhere A, Manke T, Vingron M (2007) Predicting transcription factor affinities to DNA from a biophysical model. Bioinformatics 23: 134-141.

68. Thomas-Chollier M, Hufton A, Heinig M, O'Keeffe S, Masri NE, et al. (2011) Transcription factor binding predictions using TRAP for the analysis of ChIPseq data and regulatory SNPs. Nature protocols 6: 1860-1869.

69. Kohany O, Gentles AJ, Hankus L, Jurka J (2006) Annotation, submission and screening of repetitive elements in Repbase: RepbaseSubmitter and Censor. BMC bioinformatics 7: 474 .

70. Partridge NC, Frampton RJ, Eisman JA, Michelangeli VP, Elms E, et al. (1980) Receptors for 1,25( $\mathrm{OH})$-vitamin D3 enriched in cloned osteoblast-like rat osteogenic sarcoma cells. FEBS letters 115: 139-142.

71. Reese MG (2001) Application of a time-delay neural network to promoter annotation in the Drosophila melanogaster genome. Computers \& chemistry 26: $51-56$.

72. Hino J, Kangawa K, Matsuo H, Nohno T, Nishimatsu S (2004) Bone morphogenetic protein-3 family members and their biological functions. Frontiers in bioscience : a journal and virtual library 9: 1520-1529.

73. Jiang S, Chandler RL, Fritz DT, Mortlock DP, Rogers MB (2010) Repressive BMP2 gene regulatory elements near the BMP2 promoter. Biochem Biophys Res Commun 392: 124-128.

74. Lowery JW, Pazin D, Intini G, Kokabu S, Chappuis V, et al. (2011) The role of BMP2 signaling in the skeleton. Critical reviews in eukaryotic gene expression 21: $177-185$. 